\title{
Research on the temperature of the bathtub water
}

\author{
Fengli Li
}

North China Electric Power University.Huadian-Lu 689,071000 Baoding,China

18561516152@163.com

Keywords: Fundamental of Heat Transfer, finite difference method, MATLAB

\begin{abstract}
When a person fills a bathtub with hot water and settles into the bathtub,the bath gets noticeably cooler.In order to study the temperature even throughout the bathtub and as close as possible to the initial temperature, we need develop a model of the temperature of the bathtub water in space and time.First,we figure out the heat loss, then replace three-dimensional partial differential equations with two two-dimensional equations.Based on the finite difference method,we make time domain and space domain discrete, list discrete node equations by using heat balance mothod and solve discrete node equations.
\end{abstract}

\section{Introduction}

We are discussing the problem basing on the problem A in MCM 2016.

Firstly,both the person and the tub could effect the temperature,we simplify the motions made by the person with rolling motions.Secondly,counting various heat loss, by finite difference mothod,we build and solve two two-dimensional temperature models of different boundary conditions.Last,the results show the distributions of temperature are pretty proper,which proves the rationality of the two models.

\section{Developing the mathematical model}

On the basis of the fundamental of heat transfer,the three-dimensional partial differential equation of conduction heat transfer ${ }^{[1]}$, which includes the third class boundary condition,is easily to be developed.

$$
\frac{\partial t}{\partial \tau}=a\left(\frac{\partial^{2} t}{\partial x^{2}}+\frac{\partial^{2} t}{\partial y^{2}}+\frac{\partial^{2} t}{\partial z^{2}}\right)
$$

Due to the complexity of the solution of the three-dimensional partial differential equation,we replace it with two two-dimensional partial differential equations, which include the third class boundary conditions.

$$
\begin{gathered}
\text { equation II } \frac{\partial t}{\partial \tau}=a\left(\frac{\partial^{2} t}{\partial x^{2}}+\frac{\partial^{2} t}{\partial z^{2}}\right) \\
\text { boundary conditions }\left\{\begin{array}{l}
-\lambda_{1}\left(\frac{\partial t}{\partial n}\right)=h_{1}\left(t_{1}-t_{w 1}\right)+w_{1} q_{3} \\
-\lambda_{2}\left(\frac{\partial t}{\partial n}\right)=\frac{Q_{2}}{S}+w_{2} q_{3}
\end{array}\right. \\
\text { equation II } \frac{\partial t}{\partial \tau}=a\left(\frac{\partial^{2} t}{\partial x^{2}}+\frac{\partial^{2} t}{\partial y^{2}}\right) \\
\text { boundary conditions }-\lambda_{1}\left(\frac{\partial t}{\partial n}\right)=h_{1}\left(t_{1}-t_{w 1}\right)+w_{1} q_{3}
\end{gathered}
$$

By now,our work is to calculate the value of $t_{w 1}, h_{1}, Q_{2}, Q_{3}, S, a, \lambda_{1}, \lambda_{2}$. During the research,we assume the movement of the person in the bathtub is the same as rolling motion. The intensity of motion made by the person can be expressed in ${ }^{\theta_{m}}$, which effects heat transfer coefficient of te 
forced convection heat transfer by changing $N^{[2]} u_{a v}$.

$$
N_{u_{a v}}=0.01526 R_{e}^{0.082315} P_{r}^{0.4}\left(\frac{\pi r}{u T_{0}}\right)^{-0.08614} \theta_{m}^{0.10369}
$$

Here we need make a hypothesis:Temperature of inside wall of tub $t_{w 1}=39.78^{\circ} \mathrm{C}$.

\section{Prove the following:}

There is a fundamental theorem ${ }^{[3]}$ saying that the heat leaking out into to atmosphere through the bathtub is equal to the convective heat in the numerical value.We replace the leaking heat with $q_{1}$, and replace the convective heat with $q_{2}$

$t_{m 1}=\frac{t_{w 1}+t_{1}}{2} \quad, \quad R_{e}=u \times r / v \quad, \quad N_{u_{a v}}=0.01526 R_{e}^{0.082315} P_{r}^{0.4}\left(\frac{\pi r}{u T_{0}}\right)^{-0.08614} \theta_{m}^{0.10369} \quad h_{1}=\frac{\lambda}{l} N_{u_{a v}}$, $q_{1}=h_{1} \times\left(t_{1}-t_{w 1}\right) \quad$,we figure out that $\lambda_{1}=0.6265 \quad v=7.32 \times 10^{-7}\left(\mathrm{~m}^{2} / \mathrm{s}\right) \quad P_{r}=4.865$ $\alpha_{V}=3.46 \times 10^{-4}\left(K^{-1}\right)$ and $R_{e}=364188.16$.Then,assuming that $u=0.3 \mathrm{~m} / \mathrm{s} \quad T_{0}=20 \mathrm{~s} \quad \theta_{m}=15$ $r=0.4 m$,we can work out $N_{u_{a v}}=261.92 \quad h_{1}=207.90 \quad q_{1}=45.738$.

Similarly,the corresponding equations of calculating $q_{2}$ are $t_{m 2}=\frac{t_{w 2}+t_{2}}{2}, t_{w 2}=t_{w 1}-\frac{q_{1} \delta_{0}}{\lambda}, G_{r}=\frac{g \alpha_{V} \Delta t l^{3}}{v^{2}}, N_{u}=C\left(G_{r} P_{r}\right)^{n}=C R a^{n}, h_{2}=\frac{\lambda}{l} N_{u}, q_{2}=\frac{t_{1}-t_{4}}{\frac{1}{h_{1}}+\frac{1}{h_{2}}+\frac{\delta}{\lambda}} \mathrm{I}$ $\mathrm{n}$ the same manner,the result is obtained $t_{w 2}=32.56^{\circ} \mathrm{C}$ $\lambda_{2}=0.02588, P_{r}=0.7282, v=1.608 \times 10^{-5}\left(\mathrm{~m}^{2} / \mathrm{s}\right), N_{u}=108.51, G_{r}=1.96 \times 10^{9}, \delta_{0}=0.3, h_{2}=4.01$, $q_{2}=44.47$.

Comparing $q_{1}$ with $q_{2}$,we found there is no significant difference between them.We can come to the conclusion that the hypothesis is correct $t_{w 1}=39.78^{\circ} \mathrm{C}$.Besides, $h_{1}=207.90$.

$$
Q_{2}=\alpha * y *\left(0.0174 v_{f}+0.0229\right) *\left(P_{b}-P_{q}\right) * \frac{760 S}{B}
$$

The formula is used to calculate the heat loss $Q_{2}$ of the water evaporation, which leaks out into atmosphere. the temperature of bathtub water is 40 degrees, $\alpha=4.1868 / \mathrm{kcal}, y=574.2 \mathrm{kcal} / \mathrm{kg}, v_{f}=0.3 \mathrm{~m} / \mathrm{s}, P_{b}=40 \mathrm{mmH}_{g}, P_{q}=15.6 \mathrm{mmH}_{g}$, $B=761.84 \mathrm{mmH}_{g}, S=1.36 \mathrm{~m}^{2}$, we can get $Q_{2}=621.5919 \mathrm{~W}$. The method of calculating $Q_{3}$ is same as above.We can reckon $q_{3}=1414.52$ by the mothod of calculating $q_{1}$, we have calculated $\lambda_{1}=0.6265 \lambda_{2}=0.02588 h_{1}=207.90 h_{2}=4.01$. With the formula $a=\frac{\lambda}{\rho c}$, the final mathematical model can be described as

$$
\begin{aligned}
& \text { equation I } \frac{\partial t}{\partial \tau}=0.000151276\left(\frac{\partial^{2} t}{\partial x^{2}}+\frac{\partial^{2} t}{\partial z^{2}}\right) \\
& \text { boundary conditions }\left\{\begin{array}{l}
-0.6265\left(\frac{\partial t}{\partial n}\right)=207.9(40-39.78)+0.9 \times 1414.52 \\
-0.02588\left(\frac{\partial t}{\partial n}\right)=\frac{621.5919}{1.36}+0.1 \times 1414.52
\end{array}\right.
\end{aligned}
$$

equation II $\frac{\partial t}{\partial \tau}=0.000151276\left(\frac{\partial^{2} t}{\partial x^{2}}+\frac{\partial^{2} t}{\partial y^{2}}\right)$ 
boundary conditions $-0.6265\left(\frac{\partial t}{\partial n}\right)=207.9(40-39.78)+0.9 \times 1414.52$

\section{Conclusion}

On the basis of finite difference method,we need to convert the derivative terms of the mathematical model to a differential expression.In accordance with the energy conservation equation,the discrete equations of internal nodes are listed by the mothod of heat balance.

$$
\lambda \Delta y \frac{t^{i}{ }_{m-1, n}-t^{i}{ }_{m, n}}{\Delta x}+\lambda \Delta y \frac{t^{i}{ }_{m+1, n}-t^{i}{ }_{m, n}}{\Delta x}+\lambda \Delta x \frac{t^{i}{ }_{m, n+1}-t^{i}{ }_{m . n}}{\Delta y}+\lambda \Delta x \frac{t_{m, n-1}^{i}-t_{m, n}^{i}}{\Delta y}=\rho c \Delta x \Delta y \frac{t_{m, n}^{i+1}-t_{m, n}^{i}}{\Delta \tau}
$$

According to the Newton's iteration method,with the value of node $\mathrm{m}$ and its neighboring nodes at some moment,we can reckon the value of node m.

By using Matlab, in the case of selecting $\Delta x=0.0312, \Delta y=0.0378, \Delta t=0.1$, at the moment of $\tau_{1}=11 \mathrm{~min}$, the temperature distributions of the two-dimensional plane I and plane II can be simulated.

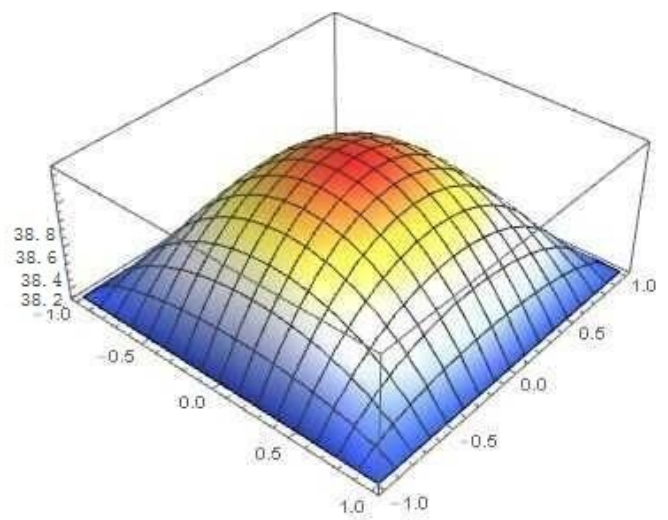

Figure 1.The Temperature Distributions of the Two-dimensional Plane I

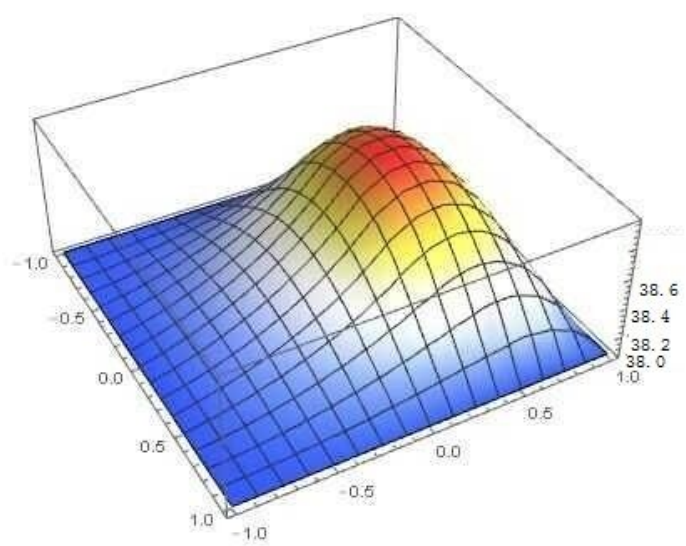

Figure 2.The Temperature Distributions of the Two-dimensional Plane II

The two-dimensional temperature distribution can reflect the temperature changes in the three-dimensional space reasonably.

\section{References}

[1]Yanfeng Liu,Zhengyang Gao.Fundamental of Heat Transfer.China Electric Power Press,2015.2.

[2]Yanming Guo,Puzhen Gao.Effects of rolling on single-phase water forced convective heat transfer characteristics.China,2009.11.

[3]Shiming Yang.Fundamental of Heat Transfer.Higher Education Press,2006. 